\title{
Trajectory-dependent electronic excitations by light and heavy ions around and below the Bohr velocity
}

\author{
S. Lohmann ๑, R. Holeňák ๑, and D. Primetzhofer \\ Department of Physics and Astronomy, Uppsala University, Box 516, 75120 Uppsala, Sweden
}

(Received 15 September 2020; accepted 10 November 2020; published 1 December 2020)

\begin{abstract}
We present experiments demonstrating trajectory-dependent electronic excitations at low ion velocities, for which ions are expected to primarily interact with delocalized valence electrons. The energy loss of $\mathrm{H}^{+}, \mathrm{H}_{2}{ }^{+}$, $\mathrm{He}^{+}, \mathrm{B}^{+}, \mathrm{N}^{+}, \mathrm{Ne}^{+},{ }^{28,29} \mathrm{Si}^{+}$, and $\mathrm{Ar}^{+}$in self-supporting silicon membranes was analyzed along channeled and random trajectories in a transmission approach. For all ions, we observe a difference in electronic stopping dependent on crystal orientation. For heavier ions, the energy-loss difference between channeling and random geometry is generally found more pronounced, and, in contrast to protons, increases for decreasing ion energy. Due to the inefficiency of core-electron excitations at employed ion velocities, we explain these results by reionization events occurring in close collisions of ions with target atoms, which are heavily suppressed for channeled trajectories. These processes result in trajectory-dependent mean charge states, which strongly affect the energy loss. The strength of the effect seems to exhibit a $Z_{1}$ oscillation with an observed minimum for Ne. We, furthermore, demonstrate that the simplicity of our experimental geometry leads to results that can serve as excellent benchmark systems for dynamic calculations of the electronic systems of solids using time-dependent density functional theory.
\end{abstract}

DOI: 10.1103/PhysRevA.102.062803

\section{INTRODUCTION}

Energy transferred by energetic charged particles to matter governs a number of astrophysical phenomena [1] and leads to radiation damage in extreme environments [2]. Detailed understanding of the energy-deposition mechanisms allows not only for predicting the mentioned effects but also for using these processes for a number of scientific and technological applications ranging from hadron therapy for cancer treatment [3] to materials characterization and modification [4]. Particularly for semiconductors, the use of ion-beam irradiations is widely employed to manipulate material properties via implantation or controlled defect creation $[5,6]$.

The energy deposition of ions is commonly denoted by the stopping power $S$, which is defined as the average energy loss per unit path length, i.e., $S=-d E / d x$. Stopping has been the subject of extensive research for decades both by modeling and experiments [7]. Many experimental studies determine $S$, in accordance with its definition, as an effective average along the ion trajectory, often by employing amorphous or polycrystalline samples. Likewise, several theoretical approaches have successfully predicted $S$ without even taking the atomic structure or electronic binding of the target material into account $[8,9]$.

Published by the American Physical Society under the terms of the Creative Commons Attribution 4.0 International license. Further distribution of this work must maintain attribution to the author(s) and the published article's title, journal citation, and DOI. Funded by Bibsam.
The individual energy-transfer events from ions to target constituents are, however, well known to be impact parameter dependent [10]. So, despite the successes of averaging approaches, the specific nature of energy deposition at the nanoscale can only be understood by going beyond them. From an experimentalist's point of view, an impact-parameter selection can be achieved by employing samples with a longrange order, i.e., single crystals. When an ion travels through a crystal with its direction of motion closely aligned with a crystal axis, it becomes subject to the channeling effect. Channeling denotes the small-angle scattering of ions by collective potentials of atomic strings at large impact parameters, leading to an oscillating motion confined to the channel [11]. At high ion energies channeling is well studied, and results in enhanced ion ranges, i.e., significantly reduced stopping, along the channel due to the suppression of core-electron excitations in close collisions (see, e.g., the reviews by Gemmell [12] and Cohen and Dauvergne [13]).

At lower ion velocities, low meaning around and below the Bohr velocity $v_{0}$, however, ion-solid interactions behave differently than at high ones, also affecting the impact-parameter dependence. Core-electron excitations become much less efficient, and the interactions with target valence electrons become nonadiabatic. A direct consequence is dynamic screening of the charge of the penetrating ion which affects its scattering probabilities. Moreover, chargeexchange events and the formation of molecular orbitals [14] directly, and indirectly via altering the mean charge state of the ion, affect the energy loss, and again the scattering potential [15]. Additionally, the structure of the target density of states has to be taken into account [16], although the influence on electronic interactions is often complex $[17,18]$. In 
particular, ions heavier than protons might significantly perturb the electronic system of the target altering the response of the system $[19,20]$.

With the development of time-dependent density functional theory (TD-DFT), a modeling tool to adequately describe these dynamic processes has become available [21]. For the sake of computational simplicity, these simulations are often done for well-defined lattices, i.e., mimicking the transmission of ions through single crystals. Several ion-target combinations have been studied this way, e.g., $\mathrm{H}$ in $\mathrm{Si}, \mathrm{Ge}$, and graphite [22-24], Si in Si [25,26], Ni in Ni [27] and W in $\mathrm{W}$ [28]; however, a comparison to adequate experimental data, i.e., data obtained under the exact same conditions using well-defined samples, is often missing.

In a previous study we have measured the energy loss of slow protons and He ions through self-supporting $\mathrm{Si}(100)$ nanomembranes both for channeling and random conditions [29]. Results for protons agree well with previous studies at higher energies, and exhibit an increase of the difference between channeled and random trajectories with ion energy due to increasing contributions of core-electron excitations for close collisions. For He ions, we observed an opposite trend, i.e., an increase of the difference between random and channeled energy loss for lower ion velocities. We explained this behavior by collision-induced charge-exchange events resulting in a higher mean charge state of the ion, at energies where the equilibrium charge state approaches zero. These charge-exchange processes lead to a direct energy loss due to electron promotion but more importantly increase the electronic stopping along random trajectories. The more complex electronic structure of heavier ions, implying the possibility for transitions involving multiple electrons plus a larger range of available charge states, can be expected to lead to even more pronounced differences. In addition, the electronic stopping of slow channeled ions has been predicted to oscillate with the projectile atomic number $Z_{1}$ by many theoretical and experimental studies [30-32]. This oscillation has also been observed specifically in single-crystalline Si [33-36], even though only one of these works is experimental, and employed samples are not well defined. For amorphous targets, $Z_{1}$ oscillations have been observed, e.g., in C [37], but the amplitude of the oscillation seems to be much reduced compared to channeling geometries. Similarly, a recent study on polycrystalline TiN showed, if at all, a very weak oscillation with $Z_{1}$, in large discrepancy with theoretical predictions by DFT [38]. A subsequent theoretical study attributed this damping of the oscillation to inhomogeneous electron densities within the TiN compound [39]. Experiments that study $Z_{1}$ oscillations for the same system and under identical conditions for random and channeling geometry have, however, not been performed in the named studies. Such a direct comparison between these two cases is expected to clarify the trajectory dependence of electronic excitations and, specifically, $Z_{1}$ oscillations.

We have, for these reasons, conducted a follow-up study to [29] investigating the energy loss of $\mathrm{B}^{+}, \mathrm{N}^{+}, \mathrm{Ne}^{+}$, ${ }^{28,29} \mathrm{Si}^{+}$, and $\mathrm{Ar}^{+}$ions with velocities $<v_{0}$ in well-defined and characterized $\mathrm{Si}(100)$ nanomembranes. We measure two-dimensional (2D) distributions of particles transmitted through the self-supporting foil targets and compare the energy deposition in channeling and random geometries to access the impact-parameter dependence of electronic excitation processes. We furthermore compare our results on self-irradiated silicon to available TD-DFT calculations.

\section{EXPERIMENTAL METHODS}

All experiments were performed using single-crystalline, self-supporting Si(100) foils (Norcada Inc. "UberFlat" silicone membranes) with nominal thicknesses between 50 and $200 \mathrm{~nm}$ as samples. Rutherford backscattering spectrometry (RBS) and time-of-flight elastic recoil detection analysis (ToF-ERDA) were employed for characterizing sample areal density and purity. For the RBS measurements, 2-MeV $\mathrm{He}^{+}$ ions provided by the 5-MV 15SDH-2 Pelletron Tandem accelerator at Uppsala University were used as probes and backscattered ions were detected at $170^{\circ}$ scattering angle with a passivated implanted planar silicon detector. To avoid channeling, incidence angles between $5^{\circ}$ and $30^{\circ}$ were chosen, and small rotations around this set value were performed by the acquisition software to further randomize the alignment. For all measurements the beam current, as measured on a gold reference, was kept below $5 \mathrm{nA}$ to avoid damaging the foils. The thinnest samples (thickness $53 \mathrm{~nm}$ ) were measured relative to a bulk gold sample. Hereby, the beam was directed onto the reference for $15 \mathrm{~s}$, then onto the sample of interest for $30 \mathrm{~s}$, then again onto the reference and so on. The number of incident particles times the detector solid angle was consequently determined for the reference and used to evaluate the $\operatorname{Si}(100)$ spectrum. Analysis to obtain areal densities was performed with help of the SIMNRA software [40]. The uncertainty of obtained results is expected to be better than $6 \%$. For samples measured by the relative method described, this uncertainty increases to about $7.5 \%$.

ToF-ERDA measurements were likewise performed with the Tandem accelerator using $36-\mathrm{MeV} \mathrm{I}^{8+}$ ions, and detecting recoiling target atoms at $45^{\circ}$ recoil angle [41]. Energy and flight time of recoils is measured in coincidence allowing for a separation of light contaminants from the $\mathrm{Si}$ matrix. Results indicate high purity in the bulk and mainly $\mathrm{H}, \mathrm{C}$, and $\mathrm{O}$ contamination in accordance with expectations of a thin surface oxide and slight contamination with hydrocarbons.

All energy-loss measurements were conducted with the time-of-flight medium energy ion scattering (ToF-MEIS) system at Uppsala University $[42,43]$. Ion beams were provided by a Danfysik implanter platform and available energies range from 20 to $350 \mathrm{keV}$ for singly charged ions. Pulsing of the beam is performed by an electrostatic chopper combined with a gating pulse resulting in pulse widths down to 1-3 ns. Several sets of horizontal and vertical slits allow for a restriction of the beam cross section to well below $(1 \times 1) \mathrm{mm}^{2}$ and a beam divergence significantly better than $0.056^{\circ}$ [for comparison, the smallest used membranes have an area of $\left.(3 \times 3) \mathrm{mm}^{2}\right]$. The current incident on the sample can consequently be reduced to 2-3 fA only. The base pressure in the experimental chamber is found to be below $1 \times 10^{-8} \mathrm{mbar}$. No specific sample cleaning has been performed on-site for the presented experiments.

We measured the energy loss of $\mathrm{H}^{+}, \mathrm{H}_{2}{ }^{+}, \mathrm{He}^{+}, \mathrm{B}^{+}, \mathrm{N}^{+}$, $\mathrm{Ne}^{+},{ }^{28,29} \mathrm{Si}^{+}$, and $\mathrm{Ar}^{+}$ions through self-supporting $\mathrm{Si}(100)$. Three different sample thicknesses were used: 53, 135, and 

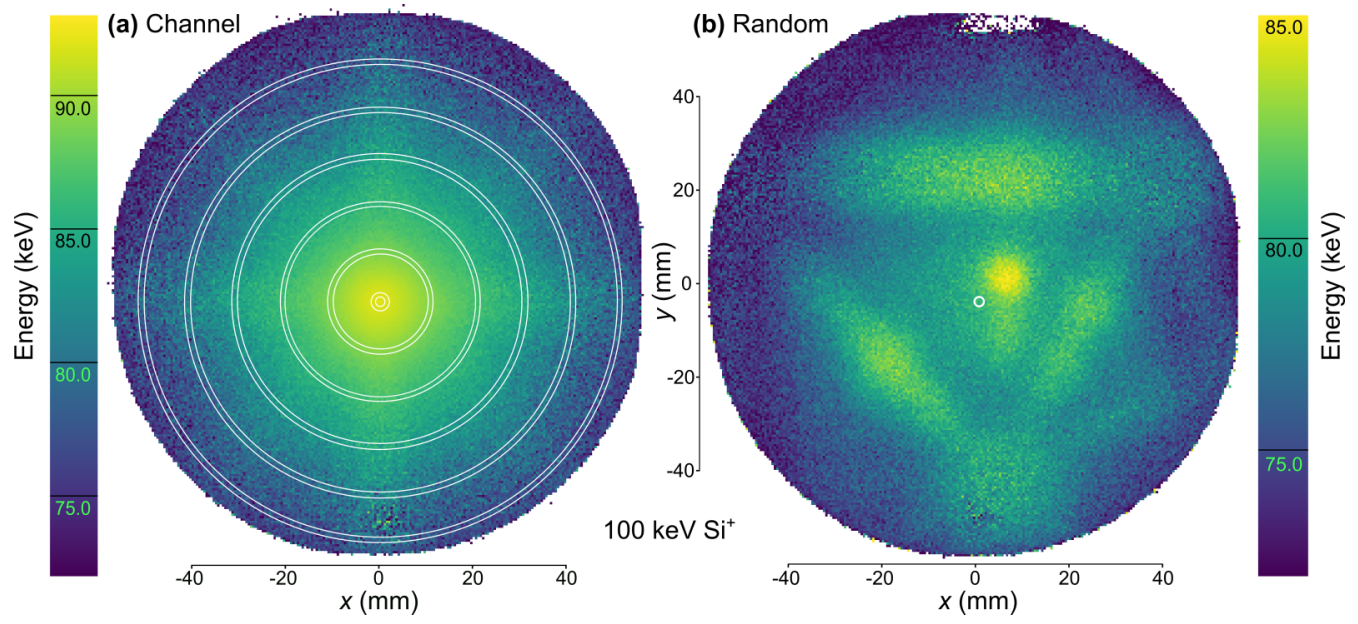

FIG. 1. Position-dependent energy of ${ }^{29} \mathrm{Si}^{+}$ions after transmission through a 53-nm-thick, self-supporting $\mathrm{Si}(100)$ foil. The initial ion energy is $100 \mathrm{keV}$, and the initial beam position is indicated by the small white circle [the innermost one in (a)]. (a) The incident beam is aligned with the principal [100] crystal axis (channeling geometry). (b) The sample has been rotated by $\theta_{x}=6^{\circ}$ around the $x$ axis and by $\theta_{y}=12^{\circ}$ around the $y$ axis arriving in a (pseudo)random position in which the beam is not aligned with any low-index channel or plane. The annuli drawn in (a) show regions of interest evaluated in Fig. 2.

$200 \mathrm{~nm}$, as obtained from the areal densities measured with RBS. Ions are transmitted through the foil, and detected $290 \mathrm{~mm}$ behind the sample with a position-sensitive microchannel plate (MCP) detector (DLD120 from RoentDek [44]). The position is determined with the help of two perpendicular delay lines, and the energy of transmitted particles is measured via their flight time. The circular detector has a diameter of $120 \mathrm{~mm}$, corresponding to deflection angles $\pm 11.5^{\circ}$, and covers a solid angle of $0.13 \mathrm{sr}$. Samples are mounted to a six-axis goniometer allowing not only for precise positioning but also for studying different beam-crystal alignments.

The ToF transmission approach together with the large size of our detector allows for 3D mapping of obtained results, i.e., a plot with information on energy and transmitted intensity available for every pixel. Details of the evaluation procedure and different available contrast modes are presented in a separate publication [45]. Figure 1 shows the energy of transmitted ions as a function of position on the detector. In both cases, ${ }^{29} \mathrm{Si}^{+}$ions with initial energies of $100 \mathrm{keV}$ are employed as probes and $\mathrm{Si}(100)$ foils with $53 \mathrm{~nm}$ thickness as samples. The projected position of the incident beam is indicated by the small white circle [the innermost in Fig. 1(a)]. The position-dependent mean energy after transmission is calculated for bins sized $(0.5 \times 0.5) \mathrm{mm}^{2}$. In Fig. 1(a), the sample is positioned in such a way that the [100] crystal axis is aligned parallel to the incident beam. In this geometry, the large majority of ions is channeled, and arrives at the detector at small scattering angles around the incident-beam position. Some ions experience scattering by larger angles, and are detected in outward regions of the detector. These ions are further influenced by the crystalline structure on their outward trajectory, i.e., they are subject to blocking and planar channeling. These effects also allow for real-space images of the crystal structure. The color contrast gives the position-dependent energies of ions after transmission, which can simply be transformed into energy-loss values by subtraction from the initial beam energy. Ions exhibit the highest detected energy, i.e., the lowest energy loss, along the chan- neled trajectory. The energy loss increases for larger scattering angles, however, planar channeling leads to energies lying between the axial channeling energy in the detector center and energies measured between the planes. This effect results in the starlike pattern visible in the figure. Note that the expected energy loss from scattering by an angle equivalent to the halfopening angle of the detector including increased trajectory length is expected to increase the energy loss by about $5 \%$.

For the measurement plotted in Fig. 1(b), the sample was rotated by $\theta_{x}=6^{\circ}$ around the $x$ axis and by $\theta_{y}=12^{\circ}$ around the $y$ axis. Then, the incident beam is no longer aligned with any low-index crystal axis, and we call this geometry "random". Otherwise, experimental conditions are the same as for the channeled incidence. The intensity of transmitted ions does not peak distinctively around the projected position of the incident beam indicating that more ions undergo scattering at larger angles. The energy distribution likewise exhibits a smaller range with a significantly lower maximum than in the channeled case. Lower energy loss in the crystal planes and high-index channels near the incident-beam direction can still be observed. Details of the energy loss will be further discussed in the following sections.

The plots presented in Fig. 1 indicate that energy-loss differences between trajectories can be assessed in two different ways: (i) different regions of interest (ROIs) on the detector corresponding to different scattering angles can be evaluated, and (ii) the sample can be rotated to compare different incidence conditions. Both methods are employed in this work. Small circular ROIs around the initial beam position (as, e.g., indicated by the innermost circle drawn in Fig. 1) can be selected to study rather straight trajectories. In order to study exclusively the electronic energy-loss contribution, the nuclear energy loss has to be considered. Even assuming suppressed nuclear stopping due to a strong trajectory selectivity in the present approach [46,47], the contribution is not fully negligible for the heaviest projectiles and a random geometry. We, therefore, performed simulations of transmission experiments using the Monte Carlo package TRBS [48] 


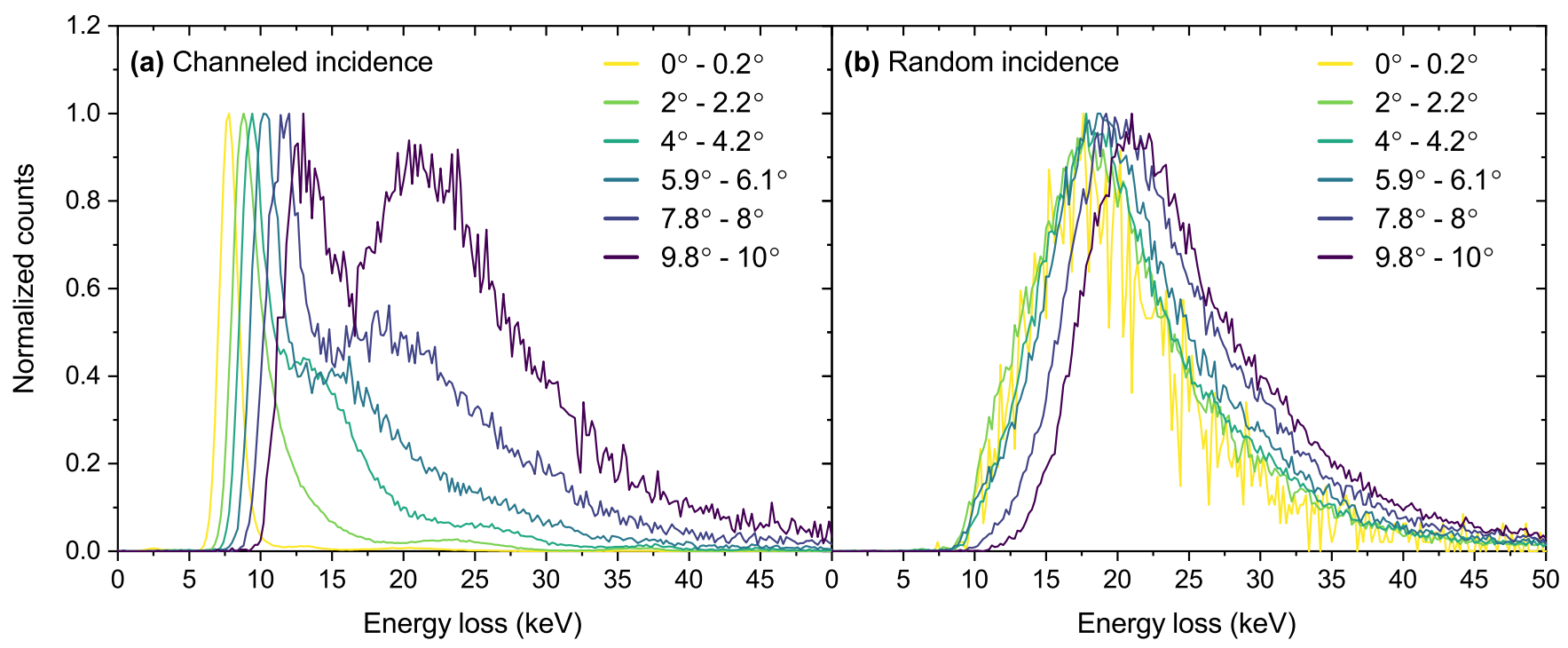

FIG. 2. Energy-loss spectra for different deflection angles recorded by transmitting ${ }^{29} \mathrm{Si}^{+}$ions with initial energy $100 \mathrm{keV}$ through a self-supporting 53-nm-thick $\mathrm{Si}(100)$ sample. The spectra in (a) (channeled incidence) and (b) (random incidence) correspond to the spatial distributions depicted in Figs. 1(a) and 1(b), respectively. Note that each figure shows data from one single measurement. The different curves are obtained by evaluating different regions of interest on the detector (annuli drawn into Fig. 1). The angular ranges given in the legend correspond to the widths of these annuli. All curves are normalized to their maximum value.

to estimate the contribution from elastic collisions. TRBS allows for modification of the Firsov screening length in the employed Thomas-Fermi-Molière potential [49]. By choosing a small screening length, the strength of the screened Coulomb potential can be decreased, and the scattering cross section drastically reduced. We performed two simulations, one with an uncorrected screening and another with the correction factor set to $1 \%$ of the uncorrected value. Whereas the first case results in simulations resembling the experiment, i.e., including potential nuclear losses, the latter scenario leads to simulations of trajectories virtually without elastic collisions. By comparing these two simulations for otherwise identical input parameters, the nuclear energy loss for a specific ion and energy can be quantified. The angular range of simulated transmitted particles was chosen to be $0-0.8^{\circ}$ to consider straight trajectories only, but still achieve sufficiently high statistics. After subtraction of the expected nuclear energy loss, the electronic stopping power can be determined from the measured energy loss employing numerical integration and assuming a $E^{1 / 2}$ dependence of the stopping power. For the narrow energy intervals, the inaccuracies introduced by a potentially different scaling are expected to be smaller than other experimental uncertainties.

\section{RESULTS}

We first evaluate energy loss as a function of detection angle for $100-\mathrm{keV}^{29} \mathrm{Si}^{+}$ions transmitted through $53-\mathrm{nm}$ $\mathrm{Si}(100)$, i.e., the measurements already presented in Fig. 1. To this aim, only ions arriving in narrow annuli centered around the incident-beam position are selected. These ROIs are also drawn in Fig. 1(a). Resulting energy-loss spectra are depicted in Fig. 2. Results for channeling and random incident geometry are compared and shown in Figs. 2(a) and 2(b), respectively. All curves are normalized to their respective maximum value for easier comparison.

Ions incident in channeled geometry and exiting at very small deflection angles [the yellow (lightest gray) curve in Fig. 2(a)] exhibit a very narrow energy distribution and the lowest energy loss of all shown cases. For larger angles, the energy loss gradually increases, and the distribution broadens. From around $4^{\circ}$ (the dark green curve) onwards, the energy-loss distribution shows two distinct peaks, which separate more clearly for larger deflection angles. For random incidence, the energy distribution is very broad even for the innermost ROI. The shape of the distribution does not change with deflection angle and only slightly shifts towards higher energy loss for larger angles. A comparison between the darkest curves in Figs. 2(a) and 2(b) shows that the high-energy-loss peak for the channeled-incidence spectrum is located at the same position as the random-incidence peak for an equivalent deflection angle of $10^{\circ}$.

A significant difference in the energy loss observed along random and channeled trajectories for the shown example becomes already apparent from Figs. 1 and 2. To study this observation more in detail and compare with our previous results for protons and He ions, we evaluate the energy loss along rather straight trajectories ending in circular ROIs with $1-\mathrm{mm}$ radius around the initial beam position (corresponding to deflection angles $\left.\pm 0.2^{\circ}\right)$ for channeled $\left(\Delta E_{\mathrm{ch}}\right)$ and random incidence $\left(\Delta E_{r}\right)$. Results for all studied ions, in the form of the ratio $\Delta E_{\mathrm{ch}} / \Delta E_{r}$ as a function of initial ion velocity [given in atomic units (a.u.)], are compiled in Fig. 3. Error bars include the time resolution of the respective measurement and the uncertainty in the flight path caused by the finite size of the beam spot and the evaluated ROI. Note that the ratio is shown for the measured energy loss, not stopping power. Due to the large difference between $\Delta E_{\mathrm{ch}}$ and $\Delta E_{r}$ and the nonlinear energy dependence of the stop- 


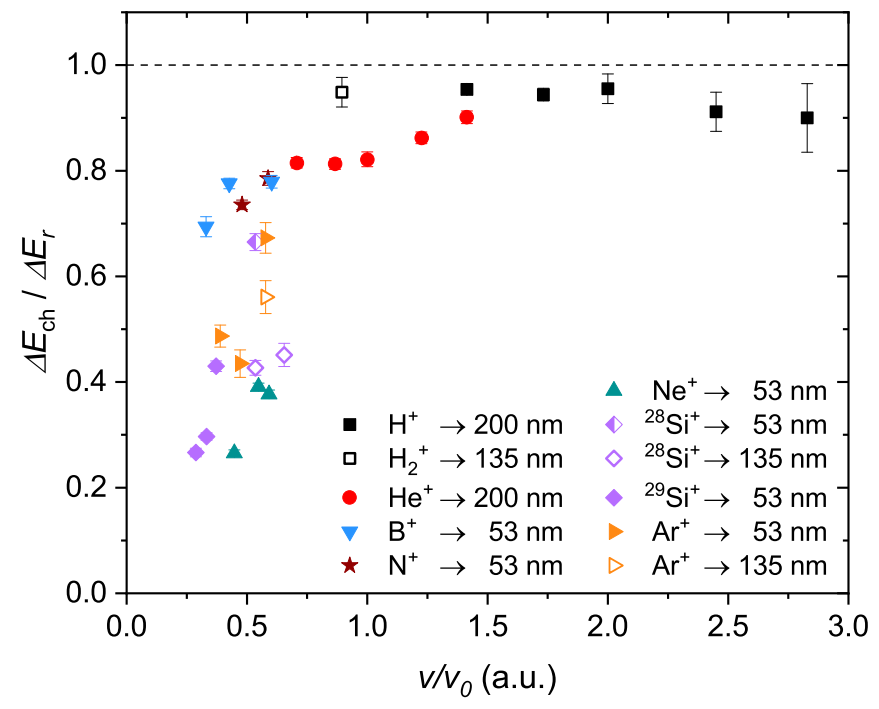

FIG. 3. Comparison between energy loss along channeled $\Delta E_{\mathrm{ch}}$ and random trajectories $\Delta E_{r}$ denoted by the fraction $\Delta E_{\mathrm{ch}} / \Delta E_{r}$ for $\mathrm{H}^{+}, \mathrm{H}_{2}{ }^{+}, \mathrm{He}^{+}, \mathrm{N}^{+}, \mathrm{Ne}^{+},{ }^{28,29} \mathrm{Si}^{+}$, and $\mathrm{Ar}^{+}$ions transmitted through self-supporting $\mathrm{Si}(100)$ membranes. Data for light ions from [29] $\left(\mathrm{H}^{+}\right.$and $\left.\mathrm{He}^{+}\right)$are shown next to ratios obtained for heavier ions with velocities well below $v_{0}$. Energy loss is evaluated along rather straight trajectories by selecting only ions that are detected in small regions of interest around the incident-beam position (for details see text).

ping power, slightly different values can be expected for the latter.

All ions heavier than protons exhibit a similar behaviora strong deviation of $\Delta E_{\mathrm{ch}} / \Delta E_{r}$ from unity and mostly a decrease of the ratio for lower velocities. Whereas for the lowest studied He velocity the channeled energy loss reaches about 0.82 of the random one, much larger differences are observed for heavier ions. As an example, $\Delta E_{\text {ch }}$ at the lowest studied velocity corresponding to $60-\mathrm{keV}$ Si reaches only 0.27 of $\Delta E_{r}$. The difference does not gradually increase with $Z_{1}$, however. Instead an apparent $Z_{1}$ oscillation is observed with the $\Delta E_{\mathrm{ch}} / \Delta E_{r}$ ratio first decreasing from He over $\mathrm{N}$ to $\mathrm{Ne}$ and then rising again over $\mathrm{Si}$ to Ar, when comparing data recorded at similar ion velocities. A complete quantification of this effect is hampered by large deviations between different data points both for Si and Ar though.

\section{DISCUSSION AND CONCLUSIONS}

The differences in energy loss for random incidence but different deflection angles can be well explained by different path length through the sample and additional kinematic losses in a single-scattering event with the respective scattering angle. For example, SIMNRA simulations for $\mathrm{Si}$ in $\mathrm{Si}$ exhibit a difference of $3 \mathrm{keV}$ between a scattering angle of close to $0^{\circ}$ and $10^{\circ}$, in good agreement with our observations. The double peak structure for channeled incidence needs an additional explanation though. The comparison with the random incidence curve at the largest studied deflection angle indicates that the peak structure featuring a high-energy loss consists of ions that have traveled on completely random trajectories, i.e., have been dechanneled very close to the sample entry point. Ions with lower energy loss must have been dechanneled later, i.e., their trajectories comprise channeled parts of various lengths. The clear separation between the two peaks indicates that the dechanneling probability is not constant over the channel length. We interpret this feature as an increased probability for dechanneling close to both surfaces, in particular in both surface oxides. Note that even a surface reconstruction, as commonly observed for clean $\mathrm{Si}(100)$, would lead to a similar process [50], although the magnitude of the effect is expected to differ between the two cases. In other words, a large fraction of ions detected at larger deflection angles is dechanneled when entering the crystal (high-energy loss) and another large fraction when exiting (low-energy loss). Figure 1(a) also indicates the occurrence of planar channeling, which would constitute another trajectory with an expected energy loss between the channeled and the random one. The broadening of the distribution suggests a mix of different trajectories ending in the same ROI on the detector. This interpretation, together with the high contrasts of observed blocking patterns and the results obtained from ToF-ERDA measurements, also confirms the high purity of and lack of lattice distortions in the crystalline bulk of employed samples.

Finally, we want to compare our results to predictions from $a b$ initio calculations. Specifically, we compare our data set for Si projectiles with TD-DFT calculations from [25]. The results are shown in Fig. 4. Purple open diamonds denote electronic stopping powers obtained in channeling geometry, whereas red filled diamonds correspond to a random beam-crystal alignment. The electronic stopping power was determined according to the procedure described in Sec. II including the subtraction of nuclear energy loss for random geometry. For the case at hand, elastic losses were found to contribute between $23 \%$ (lowest energy) and $4 \%$ (highest energy). The figure, furthermore, shows predictions from SRIM (dashed line) and an experimental data point from [52] (obtained in pseudorandom geometry) corresponding to the lowest energy data available in the IAEA stopping power database at the time of writing. The open asterisk denotes electronic stopping measured along the [110] axis from [33]. Our data measured in channeling geometry and the TD-DFT calculation that also models the passage of $\mathrm{Si}$ ions along the [100] axis of a Si crystal show excellent agreement. Electronic stopping powers along random trajectories are well predicted by SRIM. The scatter of data points at a velocity of 0.55 in Fig. 4 is expected to result from an inaccuracy or time evolution in the beam alignment during experiments but still features good agreement with predictions on average. Note that even though the relative discrepancy between channeling and random, as visible in Fig. 3, decreases, the absolute one increases with ion velocity.

For slow heavy ions, critical angles for axial and planar channeling are expected to be much larger than for $\mathrm{H}$ and $\mathrm{He}$. Thus, finding a completely random alignment of the beam for the employed samples is experimentally not straightforward. As an example, in Fig. 1(b) channeling in a high-index channel close to the incident-beam position is observed (the high-energy region at the top-right of the white circle). In this context, as mentioned above, in Fig. 4, intercomparing 


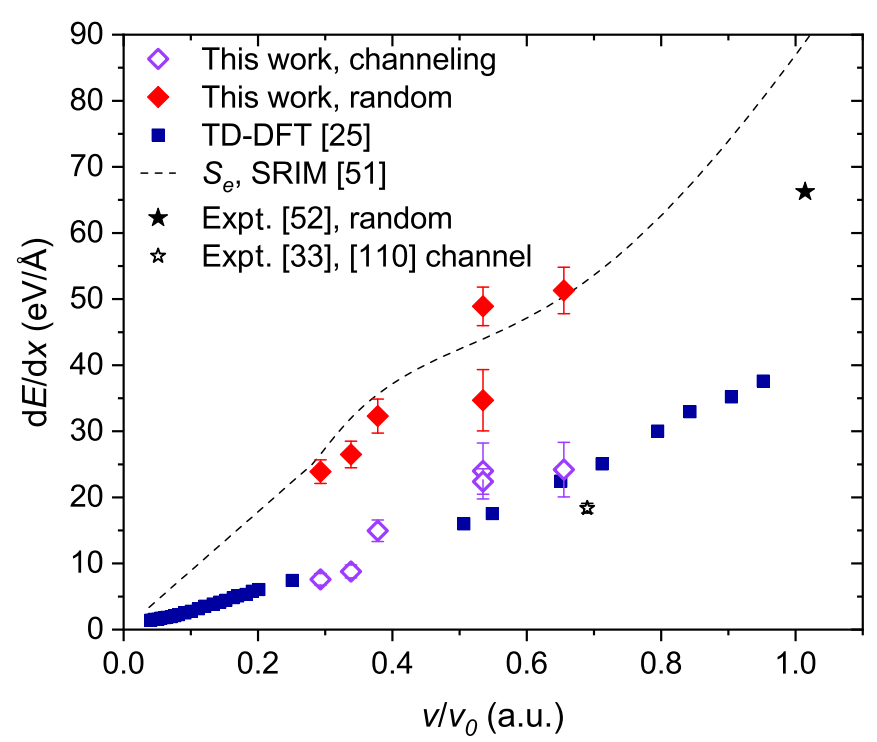

FIG. 4. Comparison of experimental electronic stopping powers of $\mathrm{Si}$ in $\mathrm{Si}$ with data from TD-DFT. The energy loss of ${ }^{28,29} \mathrm{Si}^{+}$ was measured in transmission through self-supporting $\mathrm{Si}(100)$ nanomembranes. For details on employed isotope-target thickness combinations see Fig. 3. Purple open diamonds represent data measured in channeling geometry whereas for red filled diamonds the sample was rotated into a random alignment with the beam. The dashed line gives the prediction from SRIM [51] and the black asterisk, measured by Arstila [52], denotes the data point at the lowest energy available in the IAEA stopping power database at the time of writing. The black open asterisk denotes experimental data from [33] measured in $\mathrm{Si}(110)$, however. TD-DFT calculations (dark blue squares) performed by Lim et al. [25] also model the passage of $\mathrm{Si}$ ions along the $\mathrm{Si}[100]$ axis.

data points obtained in random geometry with each other and the SRIM data suggests that the low electronic stopping at 0.55 a.u. might not correspond to a true random stopping, but has been measured over a partially channeled trajectory. The ratios presented in Fig. 3 for ions heavier than $\mathrm{N}$ can, thus, be perceived as upper limits rather than absolute values.

Core-electron excitations at employed ion velocities are inefficient (except for protons at the highest employed velocities), and the comparison with Monte Carlo calculations shows that even for heavy projectiles, nuclear losses along random trajectories can only explain a fraction of the observed additional energy loss. Therefore, other mechanisms have to be the cause for the difference between channeled and random trajectories. In [29] we propose that in the case of He ions, repeated capture and loss processes of electrons lead to the measured higher energy loss along random trajectories. Whereas the neutralization of ions via Auger processes happens at any impact parameter, relevant reionization mechanisms can only occur in close collisions that are strongly suppressed for channeled trajectories. For one-electron processes, promotion of electron levels occurring at small impact parameters is known to reduce the He kinetic energy by about $20 \mathrm{eV}$ [53]. Two-electron processes require more energy, but are expected to be much rarer [54]. In both cases, this direct effect is not sufficient to explain the much larger observed differences even for many charge-exchange cycles. Therefore, we suggest that the major contribution to the increased energy loss along random trajectories is an increased mean charge state of the ion with an additional minor contribution from energy dissipated directly in the excitation process.

The reported results for heavier ions can be attributed to similar mechanisms as described for He. The much lower $\Delta E_{\mathrm{ch}} / \Delta E_{r}$ values are expected to be caused by dynamic processes involving several electrons and higher possible charge states. For self-irradiated Si, TD-DFT calculations by Lee et al. show indeed a strong dependence of the electronic stopping of channeled Si ions on the initial ion charge state [26]. Furthermore, different equilibrium charge states for channeling and off-channeling projectiles are reported, thus corroborating our explanation.

The observed apparent oscillatory behavior indicates a different dependence on $Z_{1}$ of the electronic stopping power along channeled and random trajectories. Whereas our available energy range does not permit measurements for the exact same velocity for all projectiles, we qualitatively compare the oscillation of the electronic stopping power with $Z_{1}$ for the data points closest to 0.6 a.u. We observe an oscillation both for random and channeling geometry, however, the latter is significantly more pronounced. The minimum for $Z_{1}=10$ $(\mathrm{Ne})$ agrees well with previous studies on ions channeled in $\mathrm{Si}$ that report a pronounced minimum in the stopping around 10 or 11 . Analyzing our results in the context of existing literature $[33,37,38]$, it can be concluded that $Z_{1}$ oscillations of electronic stopping are a consequence of the binary collisions between the nonexcited projectile with the valence and conduction electrons of the target, in accordance with predictions from theory [55]. Projectile excitations, which become important along random trajectories, seem to severely counteract this effect. With increasing equilibrium charge state, both the amplitude of the $Z_{1}$ oscillation but also the relative discrepancy between the energy loss in channeling and random trajectory is diminishing. The latter discrepancy, however, is expected to increase again at elevated energies when the contribution of core-electron excitations to the energy loss becomes substantial.

\section{SUMMARY AND OUTLOOK}

We measured the energy loss of $\mathrm{B}, \mathrm{N}, \mathrm{Ne}, \mathrm{Si}$, and $\mathrm{Ar}$ ions in $\mathrm{Si}(100)$ along channeled and random trajectories in transmission. We found a significantly reduced energy loss in channeling geometry even for these low employed velocities below the Bohr velocity. For deflected ions, this trajectory dependence leads to a noticeable splitting of the energy distribution of an initially monoenergetic channeled beam. We explained this behavior by an increased dechanneling probability at both surfaces.

In contrast to higher velocities, core-electron excitations are expected to be suppressed for all geometries. We, therefore, proposed the observed difference in energy loss to be routed in repeated electron-capture and -loss processes induced in close encounters with target nuclei available only for random trajectories. This mechanism is expected to excite the projectile and significantly raise the ion mean charge state, which leads to higher electronic stopping power. The strength of this effect depends on the projectile electronic structure, 
and we observed a drastically less pronounced oscillation with $Z_{1}$ for random geometry compared to channeled trajectories. Provided that sample surfaces are sufficiently clean, the mean charge state distribution can be accessed by measuring ion exit charge states. Experiments of this kind for different crystal orientations could, therefore, help to study the observed phenomena further.

We also showed that the simplicity of our experimental approach together with the high quality of employed samples can provide excellent benchmark data for stopping powers calculated with TD-DFT for ion velocities below the
Bohr velocity where experimental data are scarce. Nevertheless, TD-DFT codes will need to include charge-exchange mechanisms to accurately predict stopping powers even for polycrystalline and amorphous materials.

\section{ACKNOWLEDGMENTS}

Support of accelerator operation by the Swedish Research Council VR-RFI (Contract No. 2017-00646_9) and the Swedish Foundation for Strategic Research (Contract No. RIF14-0053) is gratefully acknowledged.
[1] J. L. Fox, M. I. Galand, and R. E. Johnson, Energy deposition in planetary atmospheres by charged particles and solar photons, Space Sci. Rev. 139, 3 (2008).

[2] M. Yamaguchi, Radiation-resistant solar cells for space use, Sol. Energy Mater. Sol. Cells 68, 31 (2001).

[3] G. Kraft, Tumor therapy with heavy charged particles, Prog. Part. Nucl. Phys. 45, S473 (2000).

[4] B. Schmidt and K. Wetzig, Ion Beams in Materials Processing and Analysis (Springer-Verlag, Wien, 2013).

[5] D. N. Jamieson, C. Yang, T. Hopf, S. M. Hearne, C. I. Pakes, S. Prawer, M. Mitic, E. Gauja, S. E. Andresen, F. E. Hudson, A. S. Dzurak, and R. G. Clark, Controlled shallow single-ion implantation in silicon using an active substrate for sub-20-keV ions, Appl. Phys. Lett. 86, 202101 (2005).

[6] H. Hieslmair, L. Mandrell, I. Latchford, M. Chun, J. Sullivan, and B. Adibi, High throughput ion-implantation for silicon solar cells, Ener. Procedia 27, 122 (2012).

[7] P. Sigmund, Six decades of atomic collisions in solids, Nucl. Instrum. Methods Phys. Res. B 406, 391 (2017).

[8] J. Lindhard and A. Winther, Stopping power of electron gas and equipartition rule, Mat. Fys. Medd. Dan. Vid. Selsk. 34, 4 (1964).

[9] P. M. Echenique, R. M. Nieminen, and R. H. Ritchie, Density functional calculation of stopping power of an electron gas for slow ions, Solid State Commun. 37, 779 (1981).

[10] W. Brandt, K. W. Jones, and H. W. Kraner, Impact-Parameter Dependence of K-Shell Coulomb-Ionization Cross Sections, Phys. Rev. Lett. 30, 351 (1973).

[11] J. Lindhard, Influence of crystal lattice on motion of energetic charged particles, Mat. Fys. Medd. Dan. Vid. Selsk. 34, 14 (1965).

[12] D. S. Gemmell, Channeling and related effects in the motion of charged particles through crystals, Rev. Mod. Phys. 46, 129 (1974).

[13] C. Cohen and D. Dauvergne, High energy ion channeling: Principles and typical applications, Nucl. Instrum. Methods Phys. Res. B 225, 40 (2004).

[14] P. Riccardi, A. Sindona, and C. A. Dukes, Local charge exchange of $\mathrm{He}^{+}$ions at aluminum surfaces, Phys. Lett. A 381, 1174 (2017).

[15] R. A. Wilhelm and P. L. Grande, Unraveling energy loss processes of low energy heavy ions in 2D materials, Commun. Phys. 2, 89 (2019).

[16] E. A. Figueroa, E. D. Cantero, J. C. Eckardt, G. H. Lantschner, J. E. Valdés, and N. R. Arista, Threshold effect in the energy loss of slow protons and deuterons channeled in Au crystals, Phys. Rev. A 75, 010901(R) (2007).

[17] M. Draxler, S. P. Chenakin, S. N. Markin, and P. Bauer, Apparent Velocity Threshold in the Electronic Stopping of Slow Hydrogen Ions in LiF, Phys. Rev. Lett. 95, 113201 (2005).

[18] S. N. Markin, D. Primetzhofer, and P. Bauer, Vanishing Electronic Energy Loss of Very Slow Light Ions in Insulators with Large Band Gaps, Phys. Rev. Lett. 103, 113201 (2009).

[19] D. Goebl, D. Valdés, E. Abad, R. C. Monreal, D. Primetzhofer, and P. Bauer, Band structure effects in Auger neutralization of He ions at metal surfaces, Phys. Rev. B. 84, 165428 (2011).

[20] S. Creutzburg, J. Schwestka, A. Niggas, H. Inani, M. K. Tripathi, A. George, R. Heller, R. Kozubek, L. Madauß, N. McEvoy, S. Facsko, J. Kotakoski, M. Schleberger, A. Turchanin, P. L. Grande, F. Aumayr, and R. A. Wilhelm, Vanishing influence of the band gap on the charge exchange of slow highly charged ions in freestanding single-layer $\mathrm{MoS}_{2}$, Phys. Rev. B. 102, 045408 (2020).

[21] J. M. Pruneda, D. Sánchez-Portal, A. Arnau, J. I. Juaristi, and E. Artacho, Electronic Stopping Power in LiF from First Principles, Phys. Rev. Lett. 99, 235501 (2007).

[22] D. C. Yost, Y. Yao, and Y. Kanai, Examining real-time time-dependent density functional theory nonequilibrium simulations for the calculation of electronic stopping power, Phys. Rev. B. 96, 115134 (2017).

[23] R. Ullah, F. Corsetti, D. Sánchez-Portal, and E. Artacho, Electronic stopping power in a narrow band gap semiconductor from first principles, Phys. Rev. B 91, 125203 (2015).

[24] J. Halliday and E. Artacho, Anisotropy of electronic stopping power in graphite, Phys. Rev. B 100, 104112 (2019).

[25] A. Lim, W. M. C. Foulkes, A. P. Horsfield, D. R. Mason, A. Schleife, E. W. Draeger, and A. A. Correa, Electron Elevator: Excitations across the Band Gap via a Dynamical Gap State, Phys. Rev. Lett. 116, 043201 (2016).

[26] C.-W. Lee, J. A. Stewart, R. Dingreville, S. M. Foiles, and A. Schleife, Multiscale simulations of electron and ion dynamics in self-irradiated silicon, Phys. Rev. B 102, 024107 (2020).

[27] R. Ullah, E. Artacho, and A. A. Correa, Core Electrons in the Electronic Stopping of Heavy Ions, Phys. Rev. Lett. 121, 116401 (2018).

[28] A. E. Sand, R. Ullah, and A. A. Correa, Heavy ion ranges from first-principles electron dynamics, Npj Comput. Mater. 5, 43 (2019). 
[29] S. Lohmann and D. Primetzhofer, Disparate Energy Scaling of Trajectory-Dependent Electronic Excitations for Slow Protons and He Ions, Phys. Rev. Lett. 124, 096601 (2020).

[30] L. Eriksson, J. A. Davies, and P. Jespersgaard, Range measurements in oriented tungsten single crystals (0.1-1.0 MeV). I. electronic and nuclear stopping powers, Phys. Rev. 161, 219 (1967).

[31] R. Vincent and I. Nagy, Stopping power of a degenerate electron gas for slow ions: Role of relative kinematics in $\mathrm{Z}_{1}$-oscillation below the Fermi velocity, Nucl. Instrum. Methods Phys. Res. B 256, 182 (2007).

[32] H. Winter, J. I. Juaristi, I. Nagy, A. Arnau, and P. M. Echenique, Energy loss of slow ions in a nonuniform electron gas, Phys. Rev. B 67, 245401 (2003).

[33] F. H. Eisen, Channeling of medium-mass ions through silicon, Can. J. Phys. 46, 561 (1968).

[34] Y.-N. Wang, T.-C. Ma, and Y. Gong, $Z_{1}$ oscillation in electronic stopping power for slow ions, Phys. Lett. A 167, 287 (1992).

[35] V. Hari Kumar and A. P. Pathak, $Z_{1}$ oscillations in the stopping powers of silicon and tungsten for low-velocity channelled heavy ions, J. Phys.: Condens. Matter. 5, 3163 (1993).

[36] G. Hobler and C. S. Murthy, Towards a comprehensive model of electronic stopping in amorphous and crystalline silicon, in 2000 International Conference on Ion Implantation Technology Proceedings. Ion Implantation Technology - 2000 (Cat. No.00EX432)) (IEEE, New York, 2000), pp.209-212.

[37] B. Fastrup, P. Hvelplund, and C. Sautter, Stopping cross section in carbon of $0.1-1.0 \mathrm{MeV}$ atoms with $6 \leqslant Z_{1} \leqslant 20$, Mat. Fys. Medd. Dan. Vid. Selsk. 35, 10 (1966).

[38] M. A. Sortica, V. Paneta, B. Bruckner, S. Lohmann, T. Nyberg, P. Bauer, and D. Primetzhofer, On the $\mathrm{Z}_{1}$-dependence of electronic stopping in TiN, Sci. Rep. 9, 176 (2019).

[39] F. Matias, P. L. Grande, M. Vos, P. Koval, N. E. Koval, and N. R. Arista, Nonlinear stopping effects of slow ions in a no-freeelectron system: Titanium nitride, Phys. Rev. A 100, 030701(R) (2019).

[40] M. Mayer, SIMNRA, a simulation program for the analysis of NRA, RBS and ERDA, in The Fifteenth International Conference on the Application of Accelerators in Research and Industry, edited by J. L. Duggan, B. Stippec, and I. L. Morgan, AIP Conf. Proc. No. 475 (AIP, Melville, NY, 1999), pp. 541544.

[41] Y. Zhang, H. J. Whitlow, T. Winzell, I. F. Bubb, T. Sajavaara, K. Arstila, and J. Keinonen, Detection efficiency of time-of-flight energy elastic recoil detection analysis systems, Nucl. Instrum. Methods Phys. Res. B 149, 477 (1999).

[42] M. K. Linnarsson, A. Hallén, J. Åström, D. Primetzhofer, S. Legendre, and G. Possnert, New beam line for time-of-flight medium energy ion scattering with large area position sensitive detector, Rev. Sci. Instrum. 83, 095107 (2012).

[43] M. A. Sortica, M. K. Linnarsson, D. Wessman, S. Lohmann, and D. Primetzhofer, A versatile time-of-flight medium-energy ion scattering setup using multiple delay-line detectors, Nucl. Instrum. Methods Phys. Res. B 463, 16 (2020).

[44] O. Jagutzki, V. Mergel, K. Ullmann-Pfleger, L. Spielberger, U. Spillmann, R. Dörner, and H. Schmidt-Böcking, A broadapplication microchannel-plate detector system for advanced particle or photon detection tasks: Large area imaging, precise multi-hit timing information and high detection rate, Nucl. Instrum. Methods Phys. Res. A 477, 244 (2002).

[45] R. Holeňák, S. Lohmann, and D. Primetzhofer, Contrast modes in a $3 \mathrm{D}$ ion transmission approach at $\mathrm{keV}$ energies, Ultramicroscopy 217, 113051 (2020).

[46] D. Goebl, K. Khalal-Kouache, D. Roth, E. Steinbauer, and P. Bauer, Energy loss of low-energy ions in transmission and backscattering experiments, Phys. Rev. A 88, 032901 (2013).

[47] S. R. Naqvi, G. Possnert, and D. Primetzhofer, Energy loss of slow Ne ions in Pt and Ag from TOF-MEIS and monte-carlo simulations, Nucl. Instrum. Methods Phys. Res. B 371, 76 (2016).

[48] J. P. Biersack, E. Steinbauer, and P. Bauer, A particularly fast TRIM version for ion backscattering and high energy ion implantation, Nucl. Instrum. Methods Phys. Res. B 61, 77 (1991).

[49] G. Molière, Theorie der Streuung schneller geladener Teilchen I. Einzelstreuung am abgeschirmten Coulomb-Feld, Z. Naturforsch. A 2, 133 (1947).

[50] Y. Rosandi and H. M. Urbassek, Glancing ion incidence on $\mathrm{Si}(100)$ : Influence of surface reconstruction on ion subsurface channeling, Phys. Rev. B 85, 155430 (2012).

[51] J. F. Ziegler, M. D. Ziegler, and J. P. Biersack, SRIM-The stopping and range of ions in matter, Nucl. Instrum. Methods Phys. Res. B 268, 1818 (2010).

[52] K. Arstila, An experimental method for precise determination of electronic stopping powers for heavy ions, Nucl. Instrum. Methods Phys. Res. B 168, 473 (2000).

[53] R. Souda and M. Aono, Interactions of low-energy $\mathrm{He}^{+}, \mathrm{He}^{0}$, and $\mathrm{He}^{*}$ with solid surfaces, Nucl. Instrum. Methods Phys. Res. B 15, 114 (1986).

[54] R. Souda, K. Yamamoto, W. Hayami, T. Aizawa, and Y. Ishizawa, Low-energy $\mathrm{He}$ and $\mathrm{Ne}$ scattering from $\mathrm{Al}(111)$ : reionization versus autoionization, Surf. Sci. 363, 139 (1996).

[55] P. M. Echenique, F. Flores, and R. H. Ritchie, Dynamic screening of ions in condensed matter, in Solid State Physics, edited by H. Ehrenreich and D. Turnbull (Academic Press, Cambridge, Massachusetts, 1990), pp. 229-308. 